\title{
Application of Chemically Modified and Unmodified Waste Biological Sorbents in Treatment of Wastewater
}

\author{
John Kanayochukwu Nduka \\ Environmental Chemistry and Toxicology Research Unit, Pure \& Industrial Chemistry Department, Nnamdi Azikiwe University, PMB \\ 5025, Anambra State, Awka 420001, Nigeria \\ Correspondence should be addressed to John Kanayochukwu Nduka, johnnduka2000@yahoo.co.uk
}

Received 12 October 2011; Revised 10 February 2012; Accepted 13 February 2012

Academic Editor: Licínio M. Gando-Ferreira

Copyright (๑) 2012 John Kanayochukwu Nduka. This is an open access article distributed under the Creative Commons Attribution License, which permits unrestricted use, distribution, and reproduction in any medium, provided the original work is properly cited.

\begin{abstract}
Protein wastes (feathers, goat hair) and cellulosic wastes (corn cob, coconut husks) were collected and washed with detergent solution, thoroughly rinsed and sun dried for 2 days before drying in an oven, and then ground. One-half of ground material was carbonized at a maximum temperature of $500^{\circ} \mathrm{C}$ after mixing with $\mathrm{H}_{2} \mathrm{SO}_{4}$. The carbonized parts were pulverized; both carbonized and uncarbonized sorbents were sieved into two particle sizes of 325 and $625 \mu \mathrm{m}$ using mechanical sieve. Sorbents of a given particle size were packed into glass column. Then, textile wastewater that had its physicochemical parameters previously determined was eluted into each glass column and a contact time of 60 and 120 mins was allowed before analysis. Results showed 48.15-99.98 percentage reduction of $\mathrm{NO}_{3}{ }^{-}, \mathrm{EC}, \mathrm{Cl}^{-}, \mathrm{BOD}, \mathrm{COD}, \mathrm{DO}$, TSS, and TDS, 34.67-99.93 percentage reduction of $\mathrm{NO}_{3}{ }^{-}, \mathrm{EC}, \mathrm{Cl}^{-}, \mathrm{BOD}$, COD, DO, TSS, and TDS, 52.83-97.95 percentage reduction of $\mathrm{Pb}^{2+}, \mathrm{Ni}^{2+}, \mathrm{Cr}^{3+}$ and $\mathrm{Mn}^{2+}$ and 34.59-94.87 percentage reduction of $\mathrm{Pb}^{2+}, \mathrm{Ni}^{2+}, \mathrm{Cr}^{3+}$ and $\mathrm{Mn}^{2+}$. Carbonization, small particle, size and longer contact time enhanced the sorption capabilities of the sorbents. These show that protein and cellulosic wastes can be used to detoxify wastewater.
\end{abstract}

\section{Introduction}

Wastewater may be purely domestic or may contain some industrial wastewater as well [1]. Residential wastewater is a combination of excreta, flush water, and all types of wastewater generated from household. It is more commonly known as sewage and much diluted. There are two types of domestic sewage: black water or wastewater from toilets and gray water, which is wastewater from all sources except toilets [2]. Industrial wastewater comes from commercial activities (shops, restaurants, fast food shops, hospitals, etc.), industries (e.g., chemical industries, pharmaceutical companies, textile manufacturing, etc.), agriculture (e.g., Slurry), and so forth. Wastewaters from dyeing operations are characterized by color caused by both organic and inorganic compounds [3].

The organic compounds are more problematic in industrial effluent than inorganic materials $[4,5]$ because, apart from the color, it imparts on the wastewater, biodegradation of organic material in the dye depleting the dissolved oxygen of the water thereby stressing aquatic microbes [6]. Traditional wastewater treatment technologies have shown to be ineffective for handling synthetic dyes because of the chemical stability of these pollutants [7].

There is no single or economically attractive method of treatment of textile wastewater [8], although notable achievements were made in the use of biotechnological approaches to solving the problem recently as reviewed by dos Santos et al. [9]. In addition to biological treatment, many physical and chemical treatment methods have been employed for dye removal from wastewaters such as coagulation, flocculation, filtration, oxidation or reduction, complex formation, or neutralization [10]. New adsorption/oxidation, adsorption/reduction, and many combined processes were reviewed by $\mathrm{Qu}[11]$, while radiationinduced degradation process for treatment of wastewater was reviewed by Wojnarovites and Takacs [12]. Solid sorbents have been employed in adsorption techniques to remove certain classes of chemical pollutants from waters; activated carbon is the most successfully used, but the high operating 
costs and problems with regeneration of the spent activated carbon discourage its large-scale application. Therefore, a number of nonconventional sorbents have been tried for the treatment of wastewaters, in this class are various industrial wastes, agrowaste, or natural materials available in large quantity at low cost and are classified as alternative sorbents for the removal of inorganic and organic pollutants from wastewaters [13-16]. In this present study, the aim is to determine the efficacy of chemically modified and unmodified biological (proteinous and cellulosic) waste sorbents in treatment of textile wastewater; also the effect of particle sizes, carbonization (activation), and contact time will be established.

\section{Materials and Methods}

2.1. Collection of Sorbents and Preparation. Cellulosic waste sorbents (corn cob and coconut-husk (coir)) were procured from a local market around Awka, while protein waste sorbents (goat hair and chicken feathers) were sourced from Awka main abattoir. They were thoroughly washed with soap solution, sun dried for two days before drying in an oven at $105^{\circ} \mathrm{C}$ for 2 hours, and then ground. One-half of each ground material was carbonized at a temperature of $300^{\circ} \mathrm{C}$ for $30 \mathrm{mins}$, cooled and activated with $\mathrm{H}_{2} \mathrm{SO}_{4}$, and further heated at a temperature of $500^{\circ} \mathrm{C}$ for complete carbonization. The carbonized materials were again pulverized. Both materials were sieved into two particle sizes of $325 \mu \mathrm{m}$ and $625 \mu \mathrm{m}$ using mechanical sieve.

2.2. Wastewater Collection and Analysis. Textile wastewater was collected from general cotton mills Onitsha. Physicochemical parameters of the wastewater were analyzed before and after contacting with the adsorbents for each contact time and particle size.

Nitrate was determined according to American Public Health method [17], and chemical oxygen demand (COD) was determined by the dichromate method [18]. Biological oxygen demand (BOD) and dissolved oxygen (DO) were determined electrochemically [19].

The chloride content was determined by colorimetric method, while total suspended solid (TSS) and total dissolved solid (TDS) were determined by their respective standard methods [19]. A standard $\mathrm{pH}$ meter was used to determine the $\mathrm{pH}$, and a digital conductometer (consort K120, Belgium) was used to determine electrical conductivity (EC). Heavy metals ( $\mathrm{Pb}, \mathrm{Mn}, \mathrm{Ni}$, and $\mathrm{Cr}$ ) were determined at their respective wavelengths $(281.5,278,231$, and 358$) \mathrm{nm}$ after digestion using clean filtrates of the samples by means of atomic absorption spectrophotometry $205 \mathrm{~A}$.

$2.5 \mathrm{~g}$ of each sorbent (both carbonized and uncarbonized and each particle size) were separately packed in glass adsorption column with inner diameter $11 \mathrm{~m}$, bed height $270 \mathrm{~mm}$; the wastewater was eluted into it and allowed a contact time of 60 and 120 minutes. The wastewater was collected after 60 and 120 mins, respectively, and all the previous parameters were again determined to ascertain the percentage absorption after each batch. Results are shown in Tables 1-4.

\section{Results}

From Table 1, nitrate, chemical oxygen demand (COD), biological oxygen demand (BOD), dissolved oxygen (DO), chloride $\left(\mathrm{Cl}^{-}\right)$, total suspended solids (TSS), total dissolved solid (TDS), and electrical conductivity were drastically reduced, while the $\mathrm{pH}$ was increased to near neutrality. The reduction of the parameters increased at increased contact time. Feather, either carbonized (activated) or uncarbonized was more promising at both contact time and particle size than goat hair. Smaller particle size (large surface) absorbed/adsorbed more than large particle size (smaller surface area). More reduction in concentration of pollutants also occurred at longer contact time (120 mins); the trend of absorption is for carbonized sorbent at 60 mins: $325 \mu \mathrm{m}$ feather $>325 \mu \mathrm{m}$ goat hair $>625 \mu \mathrm{m}$ feather $>625 \mu \mathrm{m}$ goat hair. For uncarbonized: $325 \mu \mathrm{m}$ feather $>325 \mu \mathrm{m}$ goat hair $>625 \mu \mathrm{m}$ feather $>625 \mu \mathrm{m}$ goat hair. At $120 \mathrm{mins}$ contact time, for carbonized, order of absorption is $325 \mu \mathrm{m}$ feather $>$ $325 \mu \mathrm{m}$ goat hair $>625 \mu \mathrm{m}$ feather $>625 \mu \mathrm{m}$ goat hair. For uncarbonized, the same trend is observed, although variations exist. The percentage range in absorption of parameters at 60 mins contact time is nitrate (96.02-99.89), COD (49.11-96.43), BOD (48.15-97.84), DO (52.00-96.67), $\mathrm{Cl}^{-}$ (56.62-88.35), TSS (99.90-99.95), TDS (99.31-99.92), and EC (93.67-95.61), while the percentage range of absorption of the same parameters at 120 mins contact time were nitrate (99.69-99.92), COD (56.96-98.21), BOD (62.96-99.07), DO (71.33-98.00), $\mathrm{Cl}^{-}$(63.49-89.13), TSS (99.95-99.98), TDS (99.29-99.93), and EC (95.00-95.92).

From Table 2, there is near equal reduction in concentration of parameters by corn cob and coconut husk; but in terms of number of parameters reduced (absorbed/ adsorbed) more, the sorption order is for carbonized at 60 mins contact time: $325 \mu \mathrm{m}$ corn cob $>325 \mu \mathrm{m}$ coconut husk $>625 \mu \mathrm{m}$ coconut husk $>625 \mu \mathrm{m}$ corn cob. For uncarbonized, $325 \mu \mathrm{m}$ corn cob $>325 \mu \mathrm{m}$ coconut husk $>625 \mu \mathrm{m}$ coconut husk $>625 \mu \mathrm{m}$ corn cob. At $120 \mathrm{mins}$ contact time, the high sorption capability of the adsorbents for the pollutants is in the order; for carbonized, $325 \mu \mathrm{m}$ corn cob $>325 \mu \mathrm{m}$ coconut husk $>625 \mu \mathrm{m}$ coconut husk $>625 \mu \mathrm{m}$ corn cob. For uncarbonized, $325 \mu \mathrm{m}$ corn cob > $325 \mu \mathrm{m}$ coconut husk $>625 \mu \mathrm{m}$ coconut husk $>625 \mu \mathrm{m}$ corn cob. The percentage ranges in absorption of the parameters at 60 mins contact time for both particle sizes are as follows; nitrate (67.35-99.29), COD (38.21-78.93), BOD (41.0581.48), DO (36.67-81.33), $\mathrm{Cl}^{-}(50.20-83.61)$, TSS (94.7697.57), TDS (97.61-99.91), and EC (87.50-89.58),while the range of percentage absorption of the parameters at 120 mins contact time for both particle size is thus; Nitrate (79.49-89.69), COD (42.86-80.36), BOD (51.85-96.30), DO (34.67-74.67), $\mathrm{Cl}^{-}$(58.61-88.84), TSS (95.14-97.60), TDS (98.34-99.93), and EC (87.92-91.46).

From Table 3, at 60 mins contact time, carbonized $325 \mu \mathrm{m}$ feather showed higher percentage absorption of 
TABLE 1: Use of carbonized/uncarbonized waste protein sorbents in removal of pollutants from wastewater.

\begin{tabular}{|c|c|c|c|c|c|c|c|c|c|c|c|c|}
\hline \multirow{2}{*}{ S/no. } & \multirow[b]{2}{*}{ Sorbents } & \multirow{2}{*}{$\begin{array}{l}\text { Particle } \\
\text { size }\end{array}$} & \multirow[b]{2}{*}{$\begin{array}{l}\text { Contact time } \\
\quad(\text { mins })\end{array}$} & \multicolumn{8}{|c|}{$\%$ absorption of physicochemical parameters } & \multirow[b]{2}{*}{$\mathrm{pH}$} \\
\hline & & & & $\mathrm{NO}_{3}{ }^{-}$ & $\mathrm{COD}$ & BOD & $\mathrm{DO}$ & Chloride & TSS & TDS & $\begin{array}{c}\text { EC. } \\
\text { (U.S/CM) }\end{array}$ & \\
\hline$(1)$ & $\begin{array}{c}\text { Carbonized goat } \\
\text { hair }\end{array}$ & $325 \mu \mathrm{m}$ & & 99.89 & 90.54 & 93.21 & 88.00 & 88.35 & 99.95 & 99.88 & 95.00 & $8.00(5.00)$ \\
\hline$(2)$ & $\begin{array}{l}\text { Carbonized } \\
\text { feather }\end{array}$ & $325 \mu \mathrm{m}$ & & 99.89 & 96.43 & 97.84 & 96.67 & 86.82 & 99.95 & 99.90 & 95.61 & $8.00(5.00)$ \\
\hline (3) & $\begin{array}{c}\text { Carbonized goat } \\
\text { hair }\end{array}$ & $625 \mu \mathrm{m}$ & 60 & 99.69 & 81.07 & 71.30 & 62.00 & 73.29 & 99.81 & 99.84 & 94.38 & $7.00(5.00)$ \\
\hline$(4)$ & $\begin{array}{l}\text { Carbonized } \\
\text { feather }\end{array}$ & $625 \mu \mathrm{m}$ & & 99.39 & 71.43 & 62.96 & 78.67 & 77.86 & 99.95 & 99.78 & 94.67 & $8.00(5.00)$ \\
\hline (5) & $\begin{array}{l}\text { Uncarbonized } \\
\text { goat hair }\end{array}$ & $325 \mu \mathrm{m}$ & & 99.49 & 75.71 & 71.60 & 81.33 & 83.79 & 99.95 & 99.31 & 93.75 & $7.00(5.00)$ \\
\hline (6) & $\begin{array}{l}\text { Uncarbonized } \\
\text { feather }\end{array}$ & $325 \mu \mathrm{m}$ & & 99.59 & 85.71 & 70.99 & 91.33 & 85.32 & 99.95 & 99.85 & 93.83 & $7.50(5.00)$ \\
\hline (7) & $\begin{array}{l}\text { Uncarbonized } \\
\text { goat hair }\end{array}$ & $625 \mu \mathrm{m}$ & & 96.02 & 49.11 & 56.79 & 52.00 & 56.62 & 99.90 & 99.92 & 93.75 & $7.50(5.00)$ \\
\hline (8) & $\begin{array}{l}\text { Uncarbonized } \\
\text { feather }\end{array}$ & $625 \mu \mathrm{m}$ & & 99.79 & 54.64 & 48.15 & 52.67 & 71.04 & 99.95 & 99.88 & 93.67 & $8.00(5.00)$ \\
\hline (9) & $\begin{array}{c}\text { Carbonized goat } \\
\text { hair }\end{array}$ & $325 \mu \mathrm{m}$ & & 99.89 & 92.68 & 96.91 & 92.00 & 89.13 & 99.95 & 99.29 & 95.50 & $8.00(5.00)$ \\
\hline (10) & $\begin{array}{l}\text { Carbonized } \\
\text { feather }\end{array}$ & $325 \mu \mathrm{m}$ & & 99.92 & 98.21 & 99.07 & 98.00 & 88.38 & 99.98 & 99.93 & 95.42 & $8.50(5.00)$ \\
\hline (11) & $\begin{array}{c}\text { Carbonized goat } \\
\text { hair }\end{array}$ & $625 \mu \mathrm{m}$ & 120 & 99.79 & 80.54 & 68.52 & 72.00 & 76.60 & 99.88 & 99.88 & 95.21 & $7.80(5.00)$ \\
\hline (12) & $\begin{array}{l}\text { Carbonized } \\
\text { feather }\end{array}$ & $625 \mu \mathrm{m}$ & & 99.79 & 80.36 & 72.84 & 92.00 & 80.35 & 99.98 & 99.85 & 95.75 & $7.80(5.00)$ \\
\hline (13) & $\begin{array}{l}\text { Uncarbonized } \\
\text { goat hair }\end{array}$ & $325 \mu \mathrm{m}$ & & 99.69 & 78.57 & 67.28 & 90.67 & 87.69 & 99.95 & 99.48 & 92.50 & $7.50(5.00)$ \\
\hline (14) & $\begin{array}{l}\text { Uncarbonized } \\
\text { feather }\end{array}$ & $325 \mu \mathrm{m}$ & & 99.80 & 88.93 & 80.86 & 94.67 & 87.11 & 99.98 & 99.88 & 95.92 & $8.00(5.00)$ \\
\hline (15) & $\begin{array}{l}\text { Uncarbonized } \\
\text { goat hair }\end{array}$ & $625 \mu \mathrm{m}$ & & 96.91 & 56.96 & 62.96 & 78.67 & 63.49 & 99.95 & 99.94 & 95.00 & $8.00(5.00)$ \\
\hline (16) & $\begin{array}{l}\text { Uncarbonized } \\
\text { feather }\end{array}$ & $625 \mu \mathrm{m}$ & & 99.90 & 58.57 & 62.96 & 71.33 & 75.55 & 99.95 & 99.93 & 95.08 & $8.00(5.00)$ \\
\hline
\end{tabular}

Value in parenthesis is $\mathrm{pH}$ result before treatment.

$\mathrm{Mn}^{2+}$ and $\mathrm{Cr}^{3+}$, while carbonized 325 goat hair showed higher percentage absorption of $\mathrm{Ni}^{2+}$. Carbonized $625 \mu \mathrm{m}$ feather had higher percentage absorption of $\mathrm{Pb}^{2+}, \mathrm{Mn}^{2+}$, and $\mathrm{Cr}^{3+}$ than carbonized $625 \mu \mathrm{m}$ goat hair which had higher percentage absorption of $\mathrm{Ni}^{2+}$. For uncarbonized, $325 \mu \mathrm{m}$ feather showed highest percentage absorption of $\mathrm{Pb}^{2+}$ and $\mathrm{Cr}^{3+}$, while $325 \mu \mathrm{m}$ goat hair absorbed highest percentage of $\mathrm{Mn}^{2+}$ and $\mathrm{Ni}^{2+}$, using $625 \mu \mathrm{m}$, feather absorbed highest percentage of $\mathrm{Mn}^{2+}$ and $\mathrm{Ni}^{2+}$, while goat hair absorbed highest percentage of $\mathrm{Pb}^{2+}$ and $\mathrm{Cr}^{3+}$.

At 120 mins contact time, carbonized $325 \mu \mathrm{m}$ feather absorbed highest percentage of $\mathrm{Pb}^{2+}, \mathrm{Mn}^{2+}$, and $\mathrm{Cr}^{3+}$, while $325 \mu \mathrm{m}$ goat hair showed highest percentage absorption of $\mathrm{Ni}^{2+}$. Using $625 \mu \mathrm{m}$, carbonized feather showed highest percentage absorption of $\mathrm{Pb}^{2+}, \mathrm{Mn}^{2+}$, and $\mathrm{Cr}^{3+}$, while goat hair showed highest percentage of $\mathrm{Ni}^{2+}$. Using uncarbonized sorbents, $325 \mu \mathrm{m}$ feather showed highest percentage absorption of $\mathrm{Pb}^{2+}, \mathrm{Cr}^{3+}$ from the solution, while $325 \mu \mathrm{m}$ goat hair removed highest percentage of $\mathrm{Mn}^{2+}$ and $\mathrm{Ni}^{2+}$. Using
$625 \mu \mathrm{m}$, goat hair had highest percentage absorption of $\mathrm{Mn}^{2+}, \mathrm{Ni}^{2+}$, and $\mathrm{Cr}^{3+}$, while feather had highest percentage absorption of $\mathrm{Pb}^{2+}$.

From Table 4, using carbonized sorbents at 60 mins contact time, $325 \mu \mathrm{m}$ corn cob showed highest percentage absorption of $\mathrm{Pb}^{2+}$ and $\mathrm{Ni}^{2+}$, while $325 \mu \mathrm{m}$ coconut husk showed highest percentage absorption of $\mathrm{Mn}^{2+}$ and $\mathrm{Cr}^{3+}$ from the wastewater. Using $625 \mu \mathrm{m}$, coconut husk absorbed highest percentage $\mathrm{Pb}^{2+}$ and $\mathrm{Cr}^{3+}$, while $625 \mu \mathrm{m}$ corn cob showed highest percentage absorption of $\mathrm{Mn}^{2+}$ and $\mathrm{Ni}^{2+}$. Using the uncarbonized at the same contact time, $325 \mu \mathrm{m}$ coconut husk had more percentage affinity for $\mathrm{Pb}^{2+}$ and $\mathrm{Cr}^{3+}$ while $325 \mu \mathrm{m}$ corn cob did so for $\mathrm{Mn}^{2+}$ and $\mathrm{Ni}^{2+}$. Using $625 \mu \mathrm{m}$, corn cob sorbed more percentage of $\mathrm{Pb}^{2+}, \mathrm{Ni}^{2+}$, and $\mathrm{Cr}^{3+}$, while $625 \mu \mathrm{m}$ coconut husk sorbed highest percentage of $\mathrm{Mn}^{2+}$.

At 120 mins contact time, carbonized $325 \mu \mathrm{m}$ corn cob sorbed highest percentage of $\mathrm{Ni}^{2+}$, while $325 \mu \mathrm{m}$ coconut husk sorbed highest percentage of $\mathrm{Pb}^{2+}, \mathrm{Mn}^{2+}$, and $\mathrm{Cr}^{3+}$. 
TABLE 2: Use of carbonized/uncarbonized waste cellulosic sorbents in removal of pollutants from wastewater.

\begin{tabular}{|c|c|c|c|c|c|c|c|c|c|c|c|c|}
\hline \multirow{2}{*}{ S/no. } & \multirow[b]{2}{*}{ Sorbents } & \multirow{2}{*}{$\begin{array}{l}\text { Particle } \\
\text { size }\end{array}$} & \multirow[b]{2}{*}{$\begin{array}{l}\text { Contact time } \\
\quad(\text { mins })\end{array}$} & \multicolumn{8}{|c|}{$\%$ absorption of physicochemical parameters } & \multirow[b]{2}{*}{$\mathrm{pH}$} \\
\hline & & & & $\mathrm{NO}_{3}{ }^{-}$ & $\mathrm{COD}$ & BOD & DO & Chloride & TSS & TDS & $\begin{array}{c}\mathrm{EC} \\
\text { (U.S./CM) } \\
\end{array}$ & \\
\hline (1) & $\begin{array}{c}\text { Carbonized } \\
\text { corn cob }\end{array}$ & $325 \mu \mathrm{m}$ & & 85.61 & 78.93 & 81.48 & 81.33 & 80.26 & 95.14 & 99.66 & 89.58 & $8.0(5.0)$ \\
\hline (2) & $\begin{array}{l}\text { Carbonized } \\
\text { coconut husk }\end{array}$ & $325 \mu \mathrm{m}$ & & 84.59 & 63.21 & 68.52 & 36.67 & 82.50 & 97.57 & 99.91 & 88.33 & $7.50(5.00)$ \\
\hline (3) & $\begin{array}{l}\text { Carbonized } \\
\text { corn cob }\end{array}$ & $625 \mu \mathrm{m}$ & 60 & 83.57 & 52.14 & 41.05 & 70.67 & 64.65 & 97.48 & 99.64 & 87.50 & $7.50(5.00)$ \\
\hline (4) & $\begin{array}{l}\text { Carbonized } \\
\text { coconut husk }\end{array}$ & $625 \mu \mathrm{m}$ & & 82.14 & 52.14 & 49.38 & 58.00 & 70.09 & 97.48 & 99.80 & 88.13 & $7.50(5.00)$ \\
\hline (5) & $\begin{array}{l}\text { Uncarbonized } \\
\text { corn cob }\end{array}$ & $325 \mu \mathrm{m}$ & & 81.02 & 78.57 & 75.31 & 54.00 & 63.01 & 97.38 & 99.37 & 88.75 & $8.00(5.00)$ \\
\hline (6) & $\begin{array}{l}\text { Uncarbonized } \\
\text { coconut husk }\end{array}$ & $325 \mu \mathrm{m}$ & & 79.29 & 62.50 & 60.49 & 48.67 & 83.61 & 95.19 & 99.82 & 88.96 & $7.50(5.00)$ \\
\hline (7) & $\begin{array}{l}\text { Uncarbonized } \\
\text { corn cob }\end{array}$ & $625 \mu \mathrm{m}$ & & 67.35 & 38.21 & 53.70 & 54.67 & 50.20 & 97.33 & 98.64 & 87.50 & $8.00(5.00)$ \\
\hline (8) & $\begin{array}{l}\text { Uncarbonized } \\
\text { coconut husk }\end{array}$ & $625 \mu \mathrm{m}$ & & 99.29 & 47.14 & 69.75 & 44.00 & 68.01 & 94.76 & 97.61 & 88.13 & $7.50(5.00)$ \\
\hline (9) & $\begin{array}{l}\text { Carbonized } \\
\text { corncob }\end{array}$ & $325 \mu \mathrm{m}$ & & 89.69 & 79.64 & 96.30 & 52.00 & 84.22 & 97.57 & 99.68 & 90.83 & $7.80(5.00)$ \\
\hline (10) & $\begin{array}{l}\text { Carbonized } \\
\text { coconut husk }\end{array}$ & $325 \mu \mathrm{m}$ & & 79.49 & 63.93 & 69.75 & 73.33 & 88.84 & 95.19 & 99.93 & 91.04 & $8.00(5.00)$ \\
\hline (11) & $\begin{array}{l}\text { Carbonized } \\
\text { corncob }\end{array}$ & $625 \mu \mathrm{m}$ & 120 & 89.50 & 58.21 & 51.85 & 74.67 & 69.77 & 97.45 & 99.68 & 90.83 & $7.50(5.00)$ \\
\hline (12) & $\begin{array}{l}\text { Carbonized } \\
\text { coconut husk }\end{array}$ & $625 \mu \mathrm{m}$ & & 89.59 & 78.57 & 70.37 & 60.00 & 73.18 & 95.14 & 99.86 & 91.25 & $7.50(5.00)$ \\
\hline (13) & $\begin{array}{l}\text { Uncarbonized } \\
\text { corn cob }\end{array}$ & $325 \mu \mathrm{m}$ & & 80.61 & 80.36 & 69.75 & 34.67 & 88.76 & 95.83 & 99.45 & 91.46 & $8.50(5.00)$ \\
\hline (14) & $\begin{array}{l}\text { Uncarbonized } \\
\text { coconut husk }\end{array}$ & $325 \mu \mathrm{m}$ & & 89.39 & 64.11 & 56.79 & 39.33 & 84.39 & 97.60 & 99.88 & 90.83 & $8.00(5.00)$ \\
\hline (15) & $\begin{array}{l}\text { Uncarbonized } \\
\text { corn cob }\end{array}$ & $625 \mu \mathrm{m}$ & & 81.02 & 42.86 & 66.05 & 46.67 & 58.61 & 97.43 & 98.98 & 87.92 & $7.50(5.00)$ \\
\hline (16) & $\begin{array}{l}\text { Uncarbonized } \\
\text { coconut husk }\end{array}$ & $625 \mu \mathrm{m}$ & & 87.14 & 56.07 & 54.94 & 58.67 & 73.18 & 97.38 & 98.33 & 88.13 & $8.00(5.00)$ \\
\hline
\end{tabular}

Value in parenthesis is $\mathrm{pH}$ result before treatment.

When using larger particle size $(625 \mu \mathrm{m})$, coconut husk sorbed highest percentage of all the metal ions examined than corn cob; using the unactivated, $325 \mu \mathrm{m}$ corn cob sorbed highest percentage of $\mathrm{Mn}^{2+}$ and $\mathrm{Ni}^{2+}$, while $325 \mu \mathrm{m}$ coconut husk sorbed highest percentage of $\mathrm{Pb}^{2+}$ and $\mathrm{Cr}^{3+}$. Using unactivated sorbent, $625 \mu \mathrm{m}$ corn cob absorbed highest percentage of $\mathrm{Ni}^{2+}$ and $\mathrm{Cr}^{3+}$, while $625 \mu \mathrm{m}$ coconut husk absorbed highest percentage of $\mathrm{Pb}^{2+}$ and $\mathrm{Mn}^{2+}$.

\section{Discussion}

Solid wastes can be advantageously used as alternative sorbents because of their low cost and local availability. Adsorption of a basic dye-methylene blue and an acidic dye-eosin on wood dust of different particle size at varying speed has been studied by monitoring color reduction [20]. Sawdust and related waste materials from timber industry that are readily available and are inexpensive sorbents have been used to remove unwanted chemical substances from waters including dyes, oils, toxic salt, and heavy metals [9, 21]. Several cellulose-based waste materials which originate from agroindustry can be used as sorbents for synthetic dyes, such include banana and orange peels [22], olive pomace [23], palm kernel fibre [24], cotton fibre [25], several others have also been used to sequestrate metals from aqueous solution [26, 27]. Since most applied materials are cellulosic, adequate attention has not been given to studies on sorption capacity of protein waste. This study has proved that protein-based waste sorbent are more efficacious in removal of pollutants from wastewater (Tables 1 and 3). This assertion is in comparison with cellulosic sorbents when placed side by side at the same particle size, activation (carbonization), and contact time, although cellulosic sorbents under study are highly efficient pollutant absorbent/adsorbent (Tables 2 and 4). A critical analysis of the results (Tables 1-4) shows high percentage reduction in the concentrations of parameters analyzed. The differences 
TABLE 3: Use of carbonized/uncarbonized waste protein sorbents in removal of heavy metals from wastewater.

\begin{tabular}{|c|c|c|c|c|c|c|c|}
\hline \multirow{2}{*}{ S/no. } & \multirow{2}{*}{ Sorbents } & \multirow{2}{*}{ Particle size } & \multirow{2}{*}{ Contact time (mins) } & \multicolumn{4}{|c|}{$\%$ absorption of heavy metals } \\
\hline & & & & $\mathrm{Pb}$ & $\mathrm{Mn}$ & $\mathrm{Ni}$ & $\mathrm{Cr}$ \\
\hline (1) & Carbonized goat hair & $325 \mu \mathrm{m}$ & \multirow{8}{*}{60} & 87.05 & 80.84 & 94.62 & 81.89 \\
\hline (2) & Carbonized feather & $325 \mu \mathrm{m}$ & & 87.05 & 83.58 & 90.51 & 92.83 \\
\hline (3) & Carbonized goat hair & $625 \mu \mathrm{m}$ & & 65.88 & 78.53 & 77.18 & 58.87 \\
\hline (4) & Carbonized feather & $625 \mu \mathrm{m}$ & & 78.82 & 79.79 & 73.33 & 73.58 \\
\hline (5) & Uncarbonized goat hair & $325 \mu \mathrm{m}$ & & 76.00 & 76.21 & 90.51 & 78.11 \\
\hline (6) & Uncarbonized feather & $325 \mu \mathrm{m}$ & & 81.18 & 74.32 & 78.97 & 83.77 \\
\hline (7) & Uncarbonized goat hair & $625 \mu \mathrm{m}$ & & 77.65 & 63.16 & 70.77 & 61.51 \\
\hline$(8)$ & Uncarbonized feather & $625 \mu \mathrm{m}$ & & 75.29 & 68.21 & 73.85 & 52.83 \\
\hline (9) & Carbonized goat hair & $325 \mu \mathrm{m}$ & \multirow{8}{*}{120} & 85.41 & 77.26 & 97.95 & 84.15 \\
\hline (10) & Carbonized feather & $325 \mu \mathrm{m}$ & & 91.53 & 82.74 & 94.36 & 89.06 \\
\hline (11) & Carbonized goat hair & $625 \mu \mathrm{m}$ & & 71.76 & 80.21 & 76.64 & 66.79 \\
\hline (12) & Carbonized feather & $625 \mu \mathrm{m}$ & & 89.18 & 80.84 & 74.87 & 76.60 \\
\hline (13) & Uncarbonized goat hair & $325 \mu \mathrm{m}$ & & 75.53 & 83.58 & 90.26 & 85.66 \\
\hline (14) & Uncarbonized feather & $325 \mu \mathrm{m}$ & & 90.12 & 81.26 & 77.69 & 92.08 \\
\hline (15) & Uncarbonized goat hair & $625 \mu \mathrm{m}$ & & 65.18 & 68.84 & 74.87 & 63.77 \\
\hline (16) & Uncarbonized feather & $625 \mu \mathrm{m}$ & & 79.76 & 68.63 & 74.62 & 63.02 \\
\hline
\end{tabular}

TABLE 4: Use of carbonized/uncarbonized waste cellulosic sorbents in removal of heavy metals from wastewater.

\begin{tabular}{|c|c|c|c|c|c|c|c|}
\hline \multirow{2}{*}{ S/no. } & \multirow{2}{*}{ Sorbents } & \multirow{2}{*}{ Particle size } & \multirow{2}{*}{ Contact time (mins) } & \multicolumn{4}{|c|}{$\%$ absorption of heavy metals } \\
\hline & & & & $\mathrm{Pb}$ & $\mathrm{Mn}$ & $\mathrm{Ni}$ & $\mathrm{Cr}$ \\
\hline (1) & Carbonized corncob & $325 \mu \mathrm{m}$ & \multirow{8}{*}{60} & 76.00 & 63.79 & 92.31 & 64.15 \\
\hline (2) & Carbonized coconut husk & $325 \mu \mathrm{m}$ & & 61.18 & 73.47 & 74.87 & 74.34 \\
\hline (3) & Carbonized corncob & $625 \mu \mathrm{m}$ & & 37.65 & 64.63 & 66.15 & 44.15 \\
\hline (4) & Carbonized coconut husk & $625 \mu \mathrm{m}$ & & 70.59 & 47.16 & 52.56 & 58.87 \\
\hline (5) & Uncarbonized corn cob & $325 \mu \mathrm{m}$ & & 66.35 & 65.68 & 76.15 & 63.02 \\
\hline (6) & Uncarbonized coconut husk & $325 \mu \mathrm{m}$ & & 75.29 & 57.47 & 63.08 & 70.19 \\
\hline (7) & Uncarbonized corn cob & $625 \mu \mathrm{m}$ & & 71.76 & 47.16 & 52.31 & 42.26 \\
\hline (8) & Uncarbonized coconut husk & $625 \mu \mathrm{m}$ & & 61.65 & 57.68 & 49.74 & 36.60 \\
\hline (9) & Carbonized corncob & $325 \mu \mathrm{m}$ & \multirow{8}{*}{120} & 71.76 & 70.11 & 94.87 & 63.40 \\
\hline (10) & Carbonized coconut husk & $325 \mu \mathrm{m}$ & & 91.06 & 77.26 & 82.56 & 83.40 \\
\hline (11) & Carbonized corncob & $625 \mu \mathrm{m}$ & & 48.24 & 78.11 & 65.13 & 52.45 \\
\hline (12) & Carbonized coconut husk & $625 \mu \mathrm{m}$ & & 74.12 & 78.11 & 68.21 & 65.28 \\
\hline (13) & Uncarbonized corn cob & $325 \mu \mathrm{m}$ & & 69.41 & 74.74 & 89.23 & 79.62 \\
\hline (14) & Uncarbonized coconut husk & $325 \mu \mathrm{m}$ & & 79.76 & 57.89 & 73.85 & 81.51 \\
\hline (15) & Uncarbonized corn cob & $625 \mu \mathrm{m}$ & & 34.59 & 47.79 & 66.15 & 50.19 \\
\hline (16) & Uncarbonized coconut husk & $625 \mu \mathrm{m}$ & & 69.41 & 57.89 & 62.05 & 50.19 \\
\hline
\end{tabular}

in the percentage absorption profiles of the sorbents for each pair of particle size at any given contact time for the parameters assessed were insignificant. The bottom line therefore is that the sorbent powders absorbed appreciable percentage concentrations of the pollutants. A number of natural features inherent in the adsorbents enable them to show high sorption capacity for the pollutants. Coconut husk and corn cob are both fibrous and granular and are with hollow internal conduits; xylem for transporting water and phloem for transporting dissolved nutrients. The pollutants are absorbed into the pits, voids, lacunae, or lumina. Activation of cellulosic adsorbents-coconut husk (coir) and corn cob causes the production of oleophilic resins which enhances high percentage absorption of the pollutants [28]. Carbonization leads to the destruction of cellulose structure in the plant materials and the size of macro-, micro-, and mesopores increases. Developed structure with larger specific surface area and porosity are obtained than in uncarbonized adsorbent, hence high percentage absorption or removal of the pollutants by carbonized sorbents than in the uncarbonized. Uncarbonized protein (feathers and goat hair) and cellulose (coir and corn cob) adsorbents have a natural waxy water proof coating with large external surface which enables them to have high percentage absorption for the pollutants. High adsorption capability is determined by large specific surface and porous structure 
of sorbents as well as by chemical interaction with the surface functional groups present in carbonized sorbents, hence high percentage removal of the pollutants by both carbonized cellulose and protein sorbents. The variations observed in the study may be as a result of adsorptiondesorption phenomenon occurring at dynamic equilibrium as adsorption may be governed by physical interaction [29]. It may also be due to agitation from wind (atmospheric phenomenon) or structural vibration. It can also be due to the fact that absorption/adsorption is nonstoichiometry and there may be inhomogeneity in the shapes, particle sizes, surface area, pore size, morphology of the sorbents, and specific retention volume for each target compounds by the sorbents. Although only textile wastewater was used in this work [30], the method can be applied to all types of waters as present result compared well with previous work in which activated and unactivated powders of rice and groundnut husk were used to remediate brewery and beverage wastewater [31]. $\mathrm{NO}_{3}{ }^{-}, \mathrm{BOD}, \mathrm{COD}$ and $\mathrm{DO}$ were analyzed to establish the presence of chromophores (group of atoms responsible for dye color as well as electron withdrawing or donating substituents that modify or intensify the color of chromophores called auxochromes). The most important chromophores are azo $(-\mathrm{N}=\mathrm{N}-)$, carbonyl $(-\mathrm{C}=\mathrm{O})$, methine $(-\mathrm{CH})$, nitro $\left(-\mathrm{NO}_{2}\right)$, and quinoid while auxochromes are amine $\left(-\mathrm{NH}_{2}\right)$, sulfonate $\left(-\mathrm{SO}_{3} \mathrm{H}\right)$, carboxyl $(-\mathrm{COOH})$, and hydroxyl $(-\mathrm{OH})[9]$. Electrical conductivities were drastically reduced by all the sorbents at all particle size, contact time, and activation following the normal trends already observed. There was also noticeable color reduction though not of interest in the present study. The decrease in the $\mathrm{pH}$ comparing with the base wastewater values shows that the active sites on the sorbents were protonated and the sorbents become positively charged; this may cause steric hinderance which may be responsible for the less percentage absorption of $\mathrm{Cr}^{3+}$ than other metal ions. This is despite the fact that $\mathrm{Cr}^{3+}$ is of lower density than other metal ions in the study, the densities are $\mathrm{Cr}^{3+}$ (7.19), $\mathrm{Ni}^{2+}$ (8.90), $\mathrm{Mn}^{2+}$ (7.42), and $\mathrm{pb}^{2+}$ (11.34) [32]. It also depends on the lone pair of electrons available on the sorbents active sites and pore size, as pore size is inversely proportional to surface area [33]. Since absorption/adsorption of pollutants by sorbents in this study cannot be attributed to any chemical interaction between the sorbents and chemical pollutants, it can be merely be attributed to weak physical adhesive forces such as van der waal, London forces as well as by mere physical entanglement/occlusion. From this work, excluding minor variation, it is established that protein sorbents have high percentage absorption capacity than cellulose but the later tend to be more abundant and therefore cost effective. In both, carbonization (activation), small particle size (large surface area), and longer contact time have overriding effect on the efficiency of biological waste sorbent in remediating wastewater; this observation is supported by other works [25, 27]. It is suggested that if three (3) adsorption glass columns are to be connected in series and the sorbents in each column is allowed a contact time of 120 mins with the wastewater before the same water is allowed entering into the next (2nd) column, and allowed another 120 mins contact time before passing into the third (3rd) column for another contact time of 120 mins, analysis of the wastewater after 360 mins ( $6 \mathrm{hrs}$ ) contact time may reveal that the wastewater is totally free of pollutants. It is therefore concluded that protein wastes (feather and goat hair) and cellulosic wastes (corn cob and coconut husk) are highly efficient in detoxification of wastewater.

\section{References}

[1] M. F. Gordon, J. C. Geten, and A. D. Okun, Water and Wastewater Engineering, John Wiley and Son, New York, NY, USA, 1986.

[2] A. Ofoefule, E. Uzodimma, and C. Ibeto, "Wastewater: treatment options and its associated benefits," in Wastewater Evaluation and Management, Fernando Sebastian Garcia Einschlag, Ed., Intech-Publishers Croatia, 2011.

[3] R. Crites and G. Techobanoglous, Small Decentralized Wastewaters Management Systems, McGraw- Hill, Singapore, 1998.

[4] J. W. Moore and E. A. Moore, Environmental Small Decentralized Wastewater Management Systems, McGraw Chemistry, Academic Press, London, UK, 1976.

[5] J. Passivirta, Chemical Ecotoxicology, Lewis Publishers, Chelsea, Mich, USA, 1991.

[6] M. Calf and C. Eddy, Wastewater Engineering, Tata McGrawHill, New Delhi, India, 3rd edition, 1991.

[7] E. Forgacs, T. Cserháti, and G. Oros, "Removal of synthetic dyes from wastewaters: a review," Environment International, vol. 30, no. 7, pp. 953-971, 2004.

[8] Y. M. Slokar and A. M. Marechal, "Methods of decoloration of textile wastewaters," Dyes and Pigments, vol. 37, no. 4, pp. 335-356, 1998.

[9] A. B. dos Santos, F. J. Cervantes, and J. B. Vanlier, "Review paper on current technologies for decoloration of textile wastewaters: perspective for anaerobic biotechnology," Bioresource Technology, vol. 918, pp. 2169-2385, 2007.

[10] P. Janos, "Non-conventional sorbents for the Dye Removal from waters: mechanisms and selected applications," in Sorbents Properties, Materials and Applications, T. P. Wills, Ed., pp. 357-381, Nova Science, New York, NY, USA, 2009.

[11] J. QU, "Research progress of novel adsorption processes in water purification: a review," Journal of Environmental Sciences, vol. 20, no. 1, pp. 1-13, 2008.

[12] L. Wojnarovites and E. Takacs, "Irradiation treatment of azodye containing wastewater. An overview," Radiation Physics and Chemistry, vol. 77, no. 3, pp. 225-244, 2008.

[13] T. A. Kurniawan, G. Y. S. Chan, W. H. Lo, and S. Babel, "Comparisons of low-cost adsorbents for treating wastewaters laden with heavy metals," Science of the Total Environment, vol. 366, no. 2-3, pp. 409-426, 2006.

[14] G. Crini, "Non-conventional low-cost adsorbents for dye removal: a review," Bioresource Technology, vol. 97, no. 9, pp. 1061-1085, 2006.

[15] S. Babel and T. A. Kurniawan, "Low-cost adsorbents for heavy metals uptake from contaminated water: a review," Journal of Hazardous Materials, vol. 97, no. 1-3, pp. 219-243, 2003.

[16] S. E. Bailey, T. J. Olin, R. M. Bricka, and D. D. Adrian, "A review of potentially low-cost sorbents for heavy metals," Water Research, vol. 33, no. 11, pp. 2469-2479, 1999.

[17] American Public Health Association, Standard Methods for the Examination of Water and Wastewater, APHA, AWWA, WEF, Washington, DC, USA, 20th edition, 1998. 
[18] F. R. Theroux, Laboratory Manual for Chemical and Bacterial Analysis of Water and Sewage, McGraw-Hill, New York, NY, USA, 3rd edition, 1943.

[19] J. Bertram and R. Balance, A Practical Guide to the Design and Implementation of Fresh Water Quality and Monitoring Programmes, United Nations Environmental Programme (UNEP) and World Health Organization (WHO). E \& FN Spon, 1996.

[20] F. N. Emengo, J. K. Nduka, C. N. Anodebe, and P. A. C. Okoye, "Dye wastewaters, alternative physiochemical treatment reagent," in Sorbents: Properties, Materials and Applications, T. P. Willis, Ed., Nova Science, New York, NY, USA, 2009.

[21] A. Shukla, Y. H. Zhang, P. Dubey, J. L. Margrave, and S. S. Shukla, "The role of sawdust in the removal of unwanted materials from water," Journal of Hazardous Materials, vol. 95, no. 1-2, pp. 137-152, 2002.

[22] G. Annadurai, S. Juang, and D. J. Lee, "Use of cellulose-based wastes for adsorption of dyes from aqueous solutions," Journal of Hazardous Materials, vol. 92, no. 3, pp. 263-274, 2002.

[23] F. Banat, S. Al-Asheh, R. Al-Ahmad, and F. Bni-Khalid, "Bench-scale and packed bed sorption of methylene blue using treated olive pomace and charcoal," Bioresource Technology, vol. 98, no. 16, pp. 3017-3025, 2007.

[24] A. E. Ofomaja, "Kinetics and mechanism of methylene blue sorption onto palm kernel fibre," Process Biochemistry, vol. 42, no. 1, pp. 16-24, 2007.

[25] M. Saleem, T. Pirzada, and R. Qadeer, "Sorption of acid violet 17 and direct red 80 dyes on cotton fiber from aqueous solutions," Colloids and Surfaces A, vol. 292, no. 2-3, pp. 246250, 2007.

[26] J. C. Igwe and A. A. Abia, "Studies on the effects of $\mathrm{pH}$ and modification of adsorbent on AS (V) removal from aqueous solution using sawdust and coconut fibre "' Chemical Society of Nigeria, vol. 32, no. 2, pp. 24-28, 2007.

[27] M. Musah, U. A. Birnin-Yauri, and A. U. Itodo, "Detoxification of $\mathrm{Pb}^{2+}$ and $\mathrm{Cr}^{3+}$ ions using derived palm kernel shell adsorbent," in Proceedings of the 34th International Conference Chemical Society of Nigeria, September 2011.

[28] Y. Kato, K. Umehara, and M. Aoyama, "An oil sorbent from wood fiber by mild pyrolysis," Holz als Roh- und Werkstoff, vol. 55, no. 6, pp. 399-401, 1997.

[29] S. Karaca, A. Gürses, and R. Bayrak, "Investigation of applicability of the various adsorption models of methylene blue adsorption onto lignite/water interface," Energy Conversion and Management, vol. 46, no. 1, pp. 33-46, 2005.

[30] E. Gallego, F. J. Roca, J. F. Perales, and X. Guardino, "Use of Sorbents in air quality control systems," in Sorbents; Properties, Materials and Applications, T. P. Wills, Ed., Nova Science, New York, NY, USA, 2009.

[31] J. K. C. Nduka, O. J. Ezeakor, and A. C. Okoye, "Characterization of wastewater and use of cellulosic waste as treatment option," Journal of Engineering Science and Technology, vol. 14, no. 11, pp. 7226-7234, 2007.

[32] G. F. Liptrot, Modern Inorganic Chemistry, Unwin Hyman, London, UK, 4th edition, 1986.

[33] E. Matisova and S. Skrabakova, "Cabon sorbents and their utilization for preconcentration of organic pollutant in environmental samples," Journal of Chromatography A, vol. 707, pp. 145-179, 1995. 

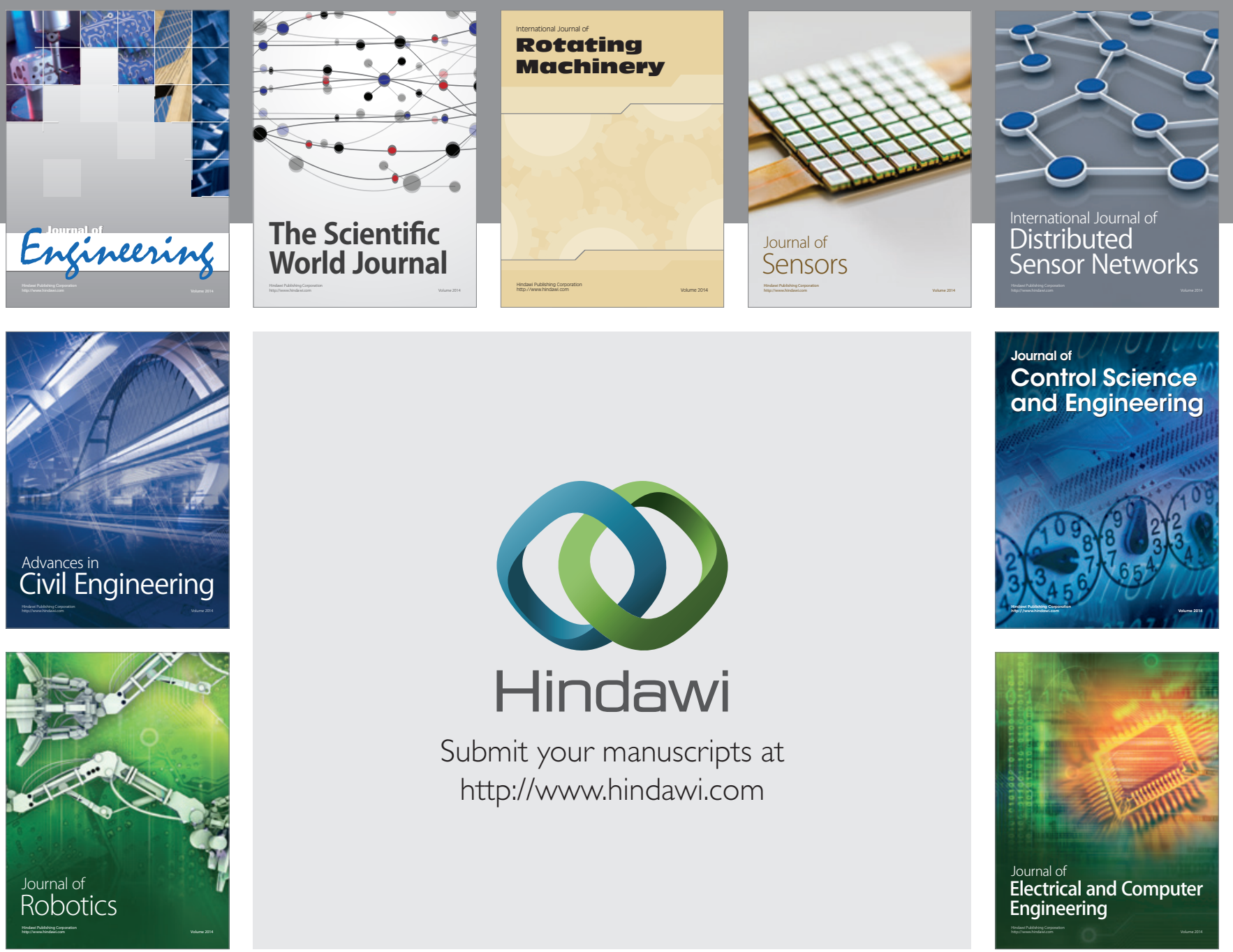

Submit your manuscripts at

http://www.hindawi.com
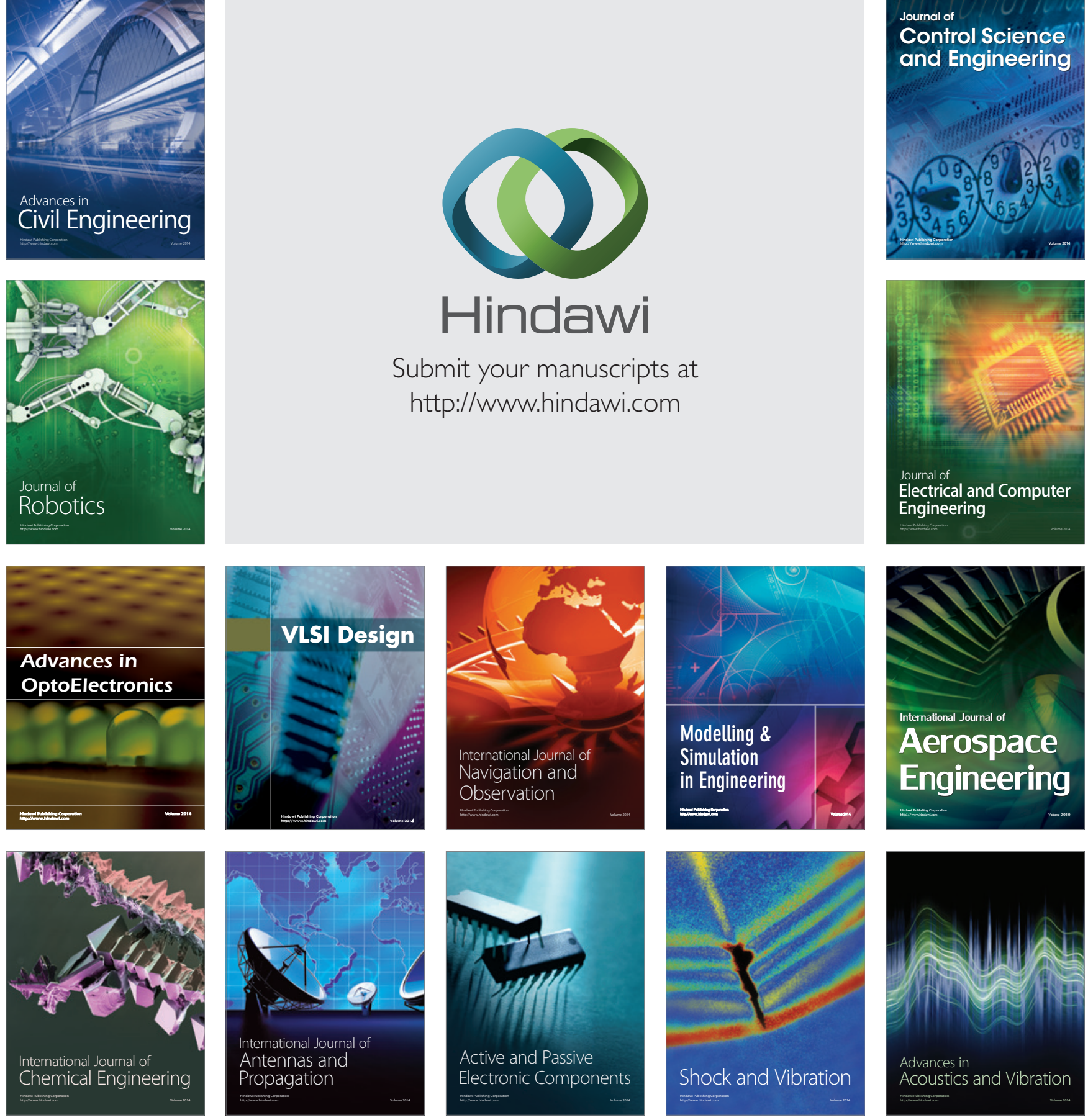